\title{
Candidate genes and potential mechanisms for chemoradiotherapy sensitivity in locally advanced rectal cancer
}

\author{
CHUNSHENG LI ${ }^{1 *}, \mathrm{CHANGYONG} \mathrm{E}^{2 *}$, YANGYANG ZHOU ${ }^{3}$ and WEI YU ${ }^{1}$ \\ Departments of ${ }^{1}$ Gastrointestinal Colorectal and Anal Surgery, and ${ }^{2}$ Hepatobiliary and Pancreatic Surgery, \\ China-Japan Union Hospital of Jilin University, Changchun, Jilin 130033; ${ }^{3}$ Department of Neurology, \\ The First Hospital of Jilin University, Changchun, Jilin 130021, P.R. China
}

Received May 29, 2018; Accepted January 15, 2019

DOI: $10.3892 / \mathrm{ol} .2019 .10087$

\begin{abstract}
The aim of the present study was to investigate candidate genes for chemoradiotherapy (CRT) sensitivity in patients with locally advanced rectal cancer (LARC), and the potential mechanisms of their action. A microarray dataset (GSE98959) was obtained from the Gene Expression Omnibus database that included microRNA (miRNA, miR) expression profiling of 22 samples from patients with LARC who had received preoperative radiotherapy and chemotherapy. Of these patients, 10 responded to the treatment and 12 did not. Differentially expressed miRNAs (DEMs) were identified, followed by the construction of an miRNA-gene network. Kyoto Encyclopedia of Genes and Genomes (KEGG) pathway and Gene Ontology (GO) function analyses were performed on the target genes in the miRNA-gene network. Furthermore, a protein-protein interaction (PPI) network was constructed on the basis of the target genes, followed by GO function enrichment and KEGG pathway analysis. A total of 30 DEMs were identified between the responder and non-responder groups. Thiamine metabolism (including miR-371a-3p) was the pathway with the highest enrichment of DEMs. The pathway that was most markedly enriched in the target genes of upregulated miRNAs was the pluripotency of stem cells pathway, as indicated by phosphoinositide-4,5-bisphosphate 3-kinase $\gamma(P I K 3 C G)$ and anaphase-promoting complex subunit 2 (APC2). Pathways in cancer exhibited the highest enrichment in the set of target genes of downregulated miRNAs. KEGG pathway and GO function analysis indicated that target genes in the PPI network were enriched in the glioma pathway and assembled in the
\end{abstract}

Correspondence to: Dr Wei Yu, Department of Gastrointestinal Colorectal and Anal Surgery, China-Japan Union Hospital of Jilin University, 126 Xiantai Street, Changchun, Jilin 130033, P.R. China E-mail: warner_y792@hotmail.com

${ }^{*}$ Contributed equally

Key words: locally advanced rectal cancer, chemoradiotherapy sensitivity, microRNA-gene regulation network, protein-protein interaction network, function and pathway analysis intracellular signaling cascade function, as indicated by the proto-oncogene NRAS. miR-371a-3p may be a candidate miRNA for CRT sensitivity in LARC via the thiamine metabolism pathway. PIK3CG and APC2 may contribute to CRT sensitivity via signaling pathways regulating the pluripotency of stem cells. Furthermore, NRAS may serve an important role in mediating CRT sensitivity via an intracellular signaling cascade.

\section{Introduction}

Locally advanced rectal cancer (LARC) occurs in the distal large intestine (1) and is the fourth most common cause of mortality worldwide, with a $\sim 30 \%$ incidence rate (2). Preoperative chemoradiotherapy (CRT) has been established as the standard treatment for LARC (3). Of patients with LARC, $12-15 \%$ undergo a complete response to long-term CRT (4). Despite CRT improving the efficiency of clinical treatment and decreasing toxicity, it does not result in an improved survival rate for patients with LARC (5). Furthermore, a number of patients with LARC do not respond well to specific CRT regimes (6). Thus, to understand the heterogeneity of patient response to CRT, investigation of predictive biomarkers in LARC is of considerable clinical interest.

MicroRNAs (miRNAs, miRs) have emerged as crucial factors in carcinogenesis (7). The translational control by miRNA expression patterns of rectal cancer can be used to predict responses to CRT (8). A previous study identified that miRNAs, including miR-215 and miR-450b-5p, are involved in the response of patients with LARC to preoperative CRT (9). Drebber et al (10) identified that high expression levels of certain miRNAs, including onco-miRNA-21, were associated with successful CRT, which further indicated an association between miRNA expression and radioresistance and chemoresistance in LARC (10). Furthermore, identifying the target genes of miRNAs is key to understanding the disease and identifying potential predictive biomarkers (11). In a study of colorectal cancer, miR-338-5p was identified to induce cancer cell migration by suppressing phosphoinositide 3-kinase subunit 3 (PIK3C3) expression and autophagy (12). Additionally, polymorphisms in miRNA-binding sites in nucleotide excision repair genes have been associated with increased risk of rectal cancer (13). However, the potential association between miRNA expression in LARC and sensitivity to CRT remains unclear. 
The miRNA expression profile of LARC samples with preoperative CRT was generated and made available in the Gene Expression Omnibus (GEO) database (GSE98959) (14). This dataset was previously used to identify differentially expressed miRNAs (DEMs) between responders and non-responders to CRT, and an association between the DEMs and $c-M Y C$ was revealed. miRNA-375 and $c-M Y C$ were suggested to be promising predictive biomarkers of the response to neoadjuvant treatment in patients with LARC (14). The aim of the present study was to identify candidate genes and key mechanisms underlying CRT sensitivity in patients with LARC, using the available miRNA expression profile dataset to investigate DEMs between responders and non-responders to CRT, and to subsequently perform a comprehensive bioinformatics analysis, including function and pathway enrichment analysis, and to conduct miRNA-target gene regulation network and a protein-protein interaction (PPI) network analysis.

\section{Materials and methods}

Microarray data. The miRNA expression profile GSE98959 dataset was obtained from the GEO database (http://www. ncbi.nlm.nih.gov/geo). In total, samples from 22 patients with LARC who had received preoperative chemotherapy and radiotherapy were profiled using TaqMan OpenArray human microRNA plates (14). Each patient sample was profiled twice, initially in pool A, and replicated in pool B.

Data preprocessing. The preprocessing of expression profile data, including original data formation, background correction and expression quantile normalization was performed in pool A and pool B using linear models for microarray data package (limma; version 3.36.1; http://www.bioconductor. org/packages/release/bioc/html/limma.html) (15) for R software (version 3.5.2; https://www.r-project.org/). The probe ID was converted into the gene symbol based on the chip platform notes file.

Analysis of DEMs. DEMs between the responder and non-responder groups were revealed respectively in pool A and pool B using the limma package. The P-values of the DEMs were corrected using Benjamini-Hochberg method (16). $\mathrm{P}<0.05$ was selected as the threshold for the identification of DEMs. Subsequently, the top 10 DEMs according to their P-values in pool A and pool B were used for further investigation.

miRNA-gene regulation network construction. Using the miRWalk 2.0 (http://zmf.umm.uni-heidelberg. de/apps/zmf/mirwalk2/index.html) (17) software, the potential target genes for the top 10 DEMs were investigated using on six databases including miRWalk (http://mirwalk.uni-hd. de) (18), miRanda (http://www.microrna.org/microrna/home. do) (19), miRDB (http://www.mirdb.org) (20), miRMap (http://mirmap.ezlab.org) (21), RNA22 (https://cm.jefferson. edu/rna22) (22) and Targetscan (http://www.targetscan. org) (23). The parameters for the miRNA information retrieval system were as follows: Minimum seed length, 7 and P-value $<0.05$. Subsequently, the common miRNA-target genes that were present in the six databases were submitted for network construction. The resulting miRNA-gene regulation network
Table I. Differentially expressed miRNAs between rectal cancer chemoradiotherapy responders and non-responders.

\begin{tabular}{ll}
\hline miRNA & P-value \\
\hline hsa-miR-381 & \\
hsa-miR-371-3p & $6.05 \times 10^{-4}$ \\
hsa-miR-644 & $2.47 \times 10^{-3}$ \\
hsa-miR-375 & $3.68 \times 10^{-3}$ \\
hsa-miR-221 & $6.27 \times 10^{-3}$ \\
hsa-miR-561 & $6.66 \times 10^{-3}$ \\
hsa-miR-1291 & $9.07 \times 10^{-3}$ \\
hsa-miR-98 & $1.00 \times 10^{-2}$ \\
hsa-miR-452\# & $1.22 \times 10^{-2}$ \\
hsa-miR-148a & $1.46 \times 10^{-2}$ \\
hsa-miR-370 & $1.59 \times 10^{-2}$ \\
hsa-miR-149\# & $1.76 \times 10^{-2}$ \\
hsa-miR-502 & $1.77 \times 10^{-2}$ \\
hsa-miR-361 & $2.05 \times 10^{-2}$ \\
hsa-miR-1272 & $2.30 \times 10^{-2}$ \\
hsa-miR-372 & $2.41 \times 10^{-2}$ \\
hsa-miR-605 & $2.55 \times 10^{-2}$ \\
hsa-miR-672 & $2.70 \times 10^{-2}$ \\
hsa-miR-888 & $2.80 \times 10^{-2}$ \\
hsa-miR-142-5p & $2.80 \times 10^{-2}$ \\
hsa-miR-155 & $3.65 \times 10^{-2}$ \\
hsa-let-7f & $3.69 \times 10^{-2}$ \\
hsa-miR-146a\# & $3.70 \times 10^{-2}$ \\
hsa-miR-129 & $4.09 \times 10^{-2}$ \\
hsa-miR-650 & $4.17 \times 10^{-2}$ \\
hsa-miR-635 & $4.29 \times 10^{-2}$ \\
hsa-miR-548K & $4.41 \times 10^{-2}$ \\
hsa-miR-617 & $4.43 \times 10^{-2}$ \\
hsa-miR-503 & $4.64 \times 10^{-2}$ \\
hsa-miR-1256 & $40^{-2}$ \\
\hline & \\
\hline
\end{tabular}

miRNA/miR, microRNA; hsa, Homo sapiens.

was visualized using cytoscape software (version 3.2.0; http://www.cytoscape.org) (24).

Functional annotation and pathway analysis. Kyoto Encyclopedia of Genes and Genomes (KEGG) pathway enrichment analysis of miRNAs in the miRNA-target gene regulation network was performed based on the clusterProfiler package (25) for R software. The Gene Ontology-Biological Process (GO-BP) function analysis and KEGG pathway analysis of target genes in the miRNA-target gene regulation network were performed by using the multifaceted analysis tool for human transcriptome (http://www.biocloudservice. com) (26). The present enrichment analyses for target genes were based on Fisher's method. $\mathrm{P}<0.01$ was considered to indicate a statistically significant difference.

PPI network construction. The search tool for the retrieval of interacting genes/proteins (STRING) database (version 10.0; 
Table II. GO function and KEGG pathway analysis for target genes associated with differentially expressed miRNAs.

\begin{tabular}{|c|c|c|c|c|c|}
\hline Analysis & Identifier & Name & Count & P-value & Genes \\
\hline \multirow[t]{5}{*}{ GO } & GO:0007242 & Intracellular signaling cascade & 7 & $4.16 \times 10^{-5}$ & NRAS, TP53, IGF1, SHC1, PAK1, PIK3R1 \\
\hline & GO:0007169 & $\begin{array}{l}\text { Transmembrane receptor protein } \\
\text { tyrosine kinase signaling pathway }\end{array}$ & 4 & $3.50 \times 10^{-4}$ & $C B L, S H C 1, P I K 3 R 1, K D R$ \\
\hline & GO:0006275 & Regulation of DNA replication & 3 & $7.29 \times 10^{-4}$ & $T P 53, I G F 1$, SHC1 \\
\hline & GO:0042127 & Regulation of cell proliferation & 5 & $1.13 \times 10^{-3}$ & NRAS, TP53, IGF1, SHC1, KDR \\
\hline & GO:0007167 & $\begin{array}{l}\text { Enzyme-linked receptor protein } \\
\text { signaling pathway }\end{array}$ & 4 & $1.20 \times 10^{-3}$ & $C B L, S H C 1, P I K 3 R 1, K D R$ \\
\hline \multirow[t]{5}{*}{ KEGG } & hsa05214 & Glioma & 6 & $3.01 \times 10^{-8}$ & PIK3CG,NRAS, TP53,IGF 1,SHCI,PIK3RI \\
\hline & hsa05220 & Chronic myeloid leukemia & 6 & $7.34 \times 10^{-8}$ & PIK3CG, NRAS, CBL, TP53, SHCl, PIK3RI \\
\hline & hsa04012 & ErbB signaling pathway & 6 & $1.56 \times 10^{-7}$ & PIK3CG, NRAS, CBL, SHC1, PAK1, PIK3R1 \\
\hline & hsa05218 & Melanoma & 5 & $4.17 \times 10^{-6}$ & PIK3CG, NRAS, TP53, IGF1, PIK3R1 \\
\hline & hsa04510 & Focal adhesion & 6 & $1.02 \times 10^{-5}$ & PIK3CG, IGF1,SHC1, PAK1, PIK3R1,KDR \\
\hline
\end{tabular}

GO, Gene Ontology; KEGG, Kyoto Encyclopedia of Genes and Genomes; miRNA, microRNA; hsa, Homo sapiens.

http://www.string-db.org) is a biological database of known and predicted PPIs (27). In the present study, STRING was used to predict interactions between the target genes of the DEMs. PPIs were selected according to the STRING database with a high confidence score of 0.7 . The centrality degree was defined as the number of connections for each target protein. The PPI network was then constructed using cytoscape software. The sub-networks (modules) with a score of $>5$ were identified using the molecular complex detection plugin (version 1.4.2; http://apps.cytoscape.org/apps/MCODE) for cytoscape. KEGG and GO-BP analysis were performed on the top 10 genes in the PPI network according to centrality degree, as well as on genes in modules with $\mathrm{P}<0.05$.

\section{Results}

Identification of DEMs. Following data preprocessing of the gene set, there were 361 and 371 miRNAs in pool A and pool $\mathrm{B}$, respectively. A total of 18 DEMs and 12 DEMs with $\mathrm{P}<0.05$ were identified in pool A and pool B, respectively. The DEMs identified in pool A and pool B were combined, with a total of 10 upregulated and 20 downregulated DEMs. These DEMs are listed in Table I.

miRNA-target gene regulation network analysis. To investigate the function of DEMs, the association between miRNAs and their target genes were identified using the following six databases: miRWalk, miRanda, miRDB, miRMap, RNA22 and Targetscan. A total of 935 interactions and 849 nodes were revealed in the miRNA-target gene interaction network. Furthermore, five upregulated miRNAs, miR-561-3p, miR-148a-5p, miR-148a-3p, miR-1291 and miR-375, and six downregulated miRNAs, miR-644a, miR-98-5p, miR-221-5p, miR-221-3p, miR-381-3p and miR-371a-3p, were prominent in the miRNA-gene regulation network (Fig. 1). Notably, the target genes of miR-371a-3p were those encoding C-terminal domain small phosphatase-like 2 , thiamin pyrophosphokinase 1 and exocyst complex component 8 .
Function and pathway investigation. KEGG pathway analysis was performed on the top 10 DEMs to evaluate their functions in the miRNA-gene regulation network. A total of 59 pathways, including thiamine metabolism [Homo sapiens (hsa) 00730; $\mathrm{P}=2.20 \times 10^{-3}$ ], signaling pathways regulating pluripotency of stem cells (hsa04550; $\mathrm{P}=6.21 \times 10^{-2}$ ) and neuroactive ligand-receptor interaction (hsa04080; $\mathrm{P}=6.71 \times 10^{-2}$ ) were enriched by DEMs. Notably, miR-371a-3p was significantly enriched in thiamine metabolism. The main pathways enriched by the top 10 DEMs are presented in Fig. 2.

GO function analysis and KEGG pathway investigation were performed on miRNA-target genes. The results of the KEGG pathway analysis indicated that genes associated with upregulated miRNAs were primarily enriched in certain pathways, including signaling pathways regulating the pluripotency of stem cells, insulin signaling pathway and Type II diabetes mellitus (Fig. 3A). The regulation of the pluripotency of stem cells pathway (hsa04550; $\mathrm{P}=2.39 \times 10^{-4}$ ) was identified by the following genes: Phosphoinositide-4,5-bisphosphate 3-kinase $\gamma(P I K 3 C G)$, SMAD family member 9, anaphase-promoting complex subunit 2 (APC2), SMAD family member 5 (SMAD5), sex-determining region Y box 2 (SOX2) and others. The insulin signaling pathway (hsa04910; $\mathrm{P}=1.06 \times 10^{-3}$ ) was identified by the genes $P I K 3 C G$, insulin receptor substrate 2 (IRS2), hexokinase domain-containing 1 (HKDC1), protein kinase AMP-activated non-catalytic subunit $\beta 2$, suppressor of cytokine signaling 1 (SOCS1) and others. The Type II diabetes mellitus pathway (hsa04930; $\mathrm{P}=5.67 \times 10^{-3}$ ) was identified by the following genes: PIK3CG, IRS2, HKDC1, SOCS1 and calcium voltage-gated channel $\alpha 1$ B. GO-BP function analysis indicated that the target genes that were associated with upregulated miRNAs were primarily enriched in the functions presented in Fig. 3B, including negative regulation of transcription by RNA polymerase II (GO, 0000122; $\mathrm{P}=8.07 \times 10^{-4}$ ) as indicated by the following genes: EP300-interacting inhibitor of differentiation 1, hepatocyte nuclear factor 1 homeobox B $(H N F 1 B)$, SATB homeo box 2, zinc finger protein $280 \mathrm{C}$, metadherin and others. 


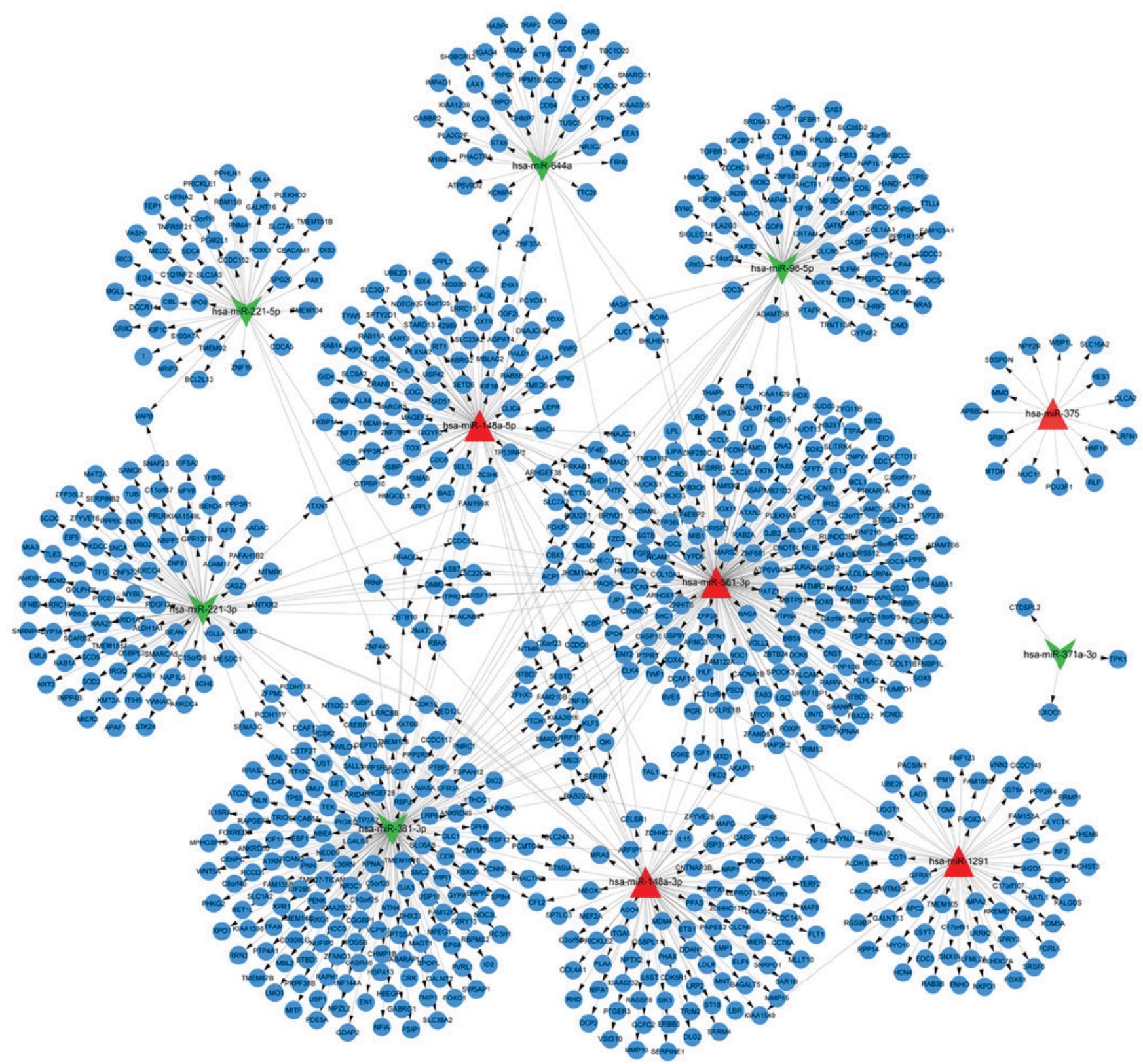

Figure 1. MicroRNA-target gene regulation network. Red triangles represent upregulated miRNAs, green arrowheads represent downregulated miRNAs, blue circles represent target genes and arrows represent the regulatory association.

Furthermore, the KEGG pathway analysis of the target genes of the downregulated miRNAs were enriched in certain pathways, including pathways in cancer (hsa05200; $\left.\mathrm{P}=2.31 \times 10^{-3}\right)$, proteoglycans in cancer (hsa05205; $\left.\mathrm{P}=2.77 \times 10^{-5}\right)$ and human T-cell lymphotropic virus-I infection (hsa05166; $\mathrm{P}=4.41 \times 10^{-3}$ ) (Fig. 3C). These three pathways were identified by the following genes: Wnt family member $5 \mathrm{~A}$, collagen type IV $\alpha 1$ chain, prostaglandin E receptor 3 (PTGER3), transforming growth factor $\beta$ receptor 1 , melanogenesis-associated transcription factor and others. The GO-BP function analysis indicated that the target genes associated with downregulated miRNAs were primarily involved in functions including positive regulation of transcription, DNA-templated (GO, 045893; $\left.\mathrm{P}=1.39 \times 10^{-3}\right)$, as indicated by the following genes: ENY2 transcription and export complex 2 subunit, HNF1B, SOX11, SMAD5, SOX2 and others (Fig. 3D).
PPI network and module analysis. To identify the potential interactions of the target genes associated with DEMs, a PPI network and associated modules were constructed on the basis of the protein interactions of the target genes. The results indicated that a total of 406 nodes and 830 interactions were included in the present PPI network (Fig. 4). According to the degree of centrality, the top 10 nodes included seven downregulated and three upregulated genes. The downregulated genes were tumor protein p53 (TP53), phosphoinositide-3-kinase regulatory subunit 1 (PIK3R1), $C B L$ proto-oncogene, insulin-like growth factor 1 (IGF1), $N R A S$, kinase insert domain receptor $(K D R)$ and p21-activated kinase $1(P A K 1)$. The three upregulated genes that were in the top 10 nodes were PIK3CG, SHC adaptor protein 1 ( $\mathrm{SHCl}$ ) and nuclear cap-binding protein subunit 1 . The GO functional analysis indicated that these genes were primarily assembled 


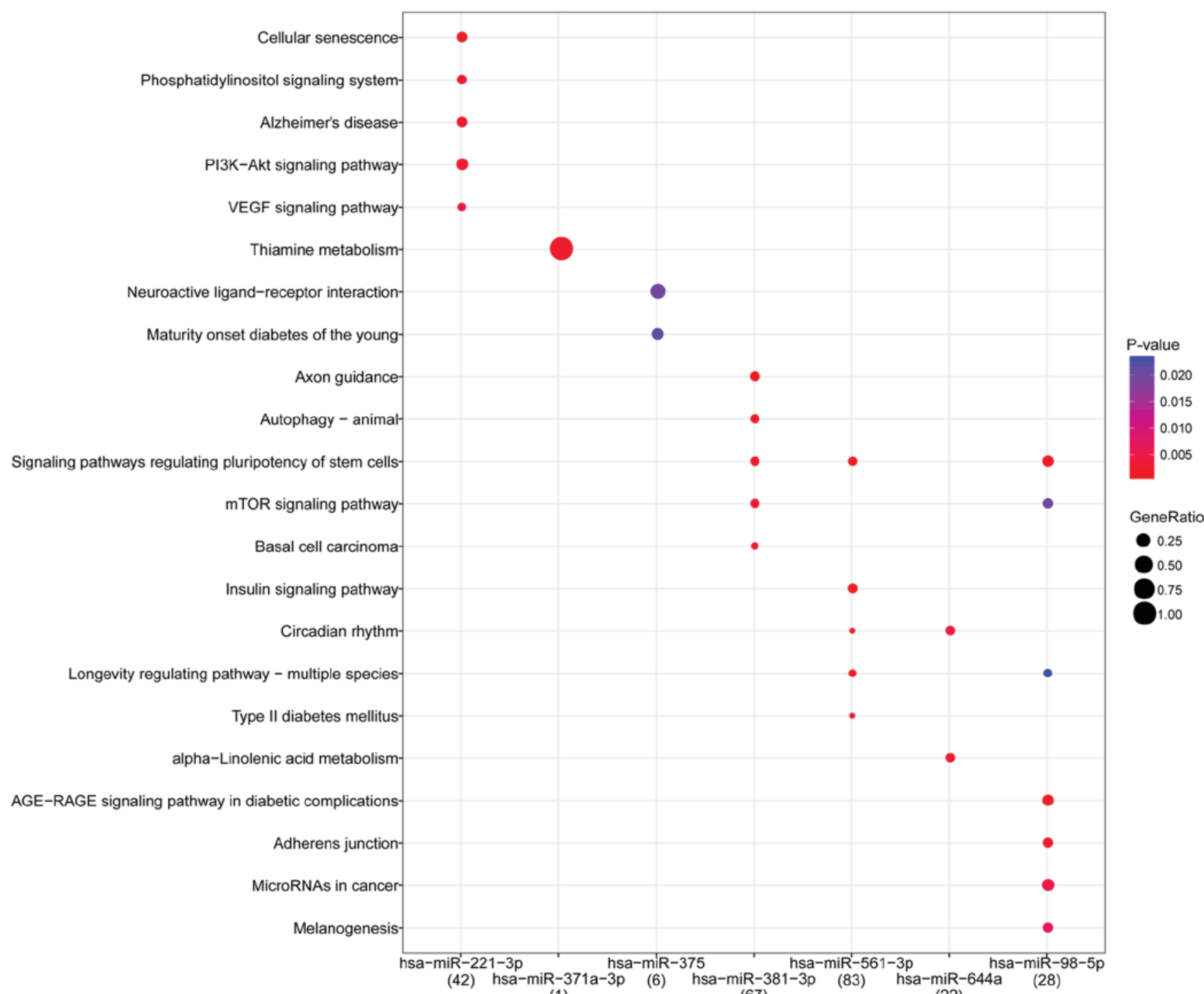

(1)

(67)

(22)

Figure 2. KEGG pathway enrichment analysis on the differentially expressed miRNAs. Darker shades of red indicate lower P-values and larger circles indicate larger gene ratios. Gene ratios are defined as the number of differentially expressed miRNAs enriched in each KEGG pathway/the total number of miRNAs enriched in all KEGG pathways. hsa, Homo sapiens; miR/miRNA, microRNA; KEGG, Kyoto Encyclopedia of Genes and Genomes.

in functions including intracellular signaling cascade $(\mathrm{GO}$, 0007242; $\left.\mathrm{P}=4.16 \times 10^{-5}\right)$, as indicated by the genes $P I K 3 C G$, NRAS, TP53, IGF1, SHC1, PAK1 and PIK3R1. The KEGG pathway analysis indicated that these genes were primarily enriched in pathways such as glioma (hsa05214; $\mathrm{P}=3.01 \times 10^{-8}$ ), as indicated by PIK3CG, NRAS, TP53, IGF1, SHCl and PIK3RI (Table II).

In addition, three modules with a score of $>5$ were identified (Fig. 5). There were 19 nodes and 82 interactions in module A (score $=9.11$ ), eight nodes and 28 interactions in module B (score $=8$ ), and 12 nodes and 29 interactions in module $\mathrm{C}$ (score=5.27). The GO functions in which the target genes of DEMs in module A, module B and module C were highly enriched were cellular macromolecule catabolic process, G-protein-coupled receptor protein signaling pathway and cell division, respectively. Cellular macromolecule catabolic process $\left(\mathrm{GO}, 0044265 ; \mathrm{P}=2.54 \times 10^{-9}\right)$, was identified by the genes E3 ubiquitin-protein ligase Praja-2, E3 ubiquitin-protein ligase RNF123, low-density lipoprotein receptor,
BTB domain-containing 1, ubiquitin-conjugating enzyme E2K $(U B E 2 K)$, and others. G-protein-coupled receptor protein signaling pathway $\left(\mathrm{GO}, 0007186 ; \mathrm{P}=2.67 \times 10^{-8}\right)$ was identified by the genes $\mathrm{P} 2 \mathrm{Y}$ purinoceptor 13 (P2RY13), sphingosine 1-phosphate receptor 1 (SIPRI), PTGER3, proenkephalin A, C-X-C motif chemokine 5 and others. Cell division (GO, 0051301; $\mathrm{P}=2.56 \times 10^{-5}$ ) was identified by the genes centromere protein O, PDS5 cohesion-associated factor B, AT-hook-containing transcription factor 1, zwilch kinetochore protein and cell division cycle-associated 5 . The KEGG pathways with the highest enrichment of target genes of DEMs in module A, module $\mathrm{B}$ and module $\mathrm{C}$ were ubiquitin-mediated proteolysis, neuroactive ligand-receptor and focal adhesion, respectively (Table III). The ubiquitin mediated proteolysis pathway (hsa04120; $\mathrm{P}=9.34 \times 10^{-5}$ ) was identified by the genes $U B E 2 K$, ubiquitin-conjugating enzyme E2 G1, SOCS1, CBL and cell division cycle 34 . The neuroactive ligand-receptor pathway (hsa04080; $\mathrm{P}=8.69 \times 10^{-5}$ ) was indicated by P2RY13, SIPRI, PTGER3, neuropeptide $\mathrm{Y}$ receptor $\mathrm{Y} 2$ and $\gamma$-aminobutyric 
A

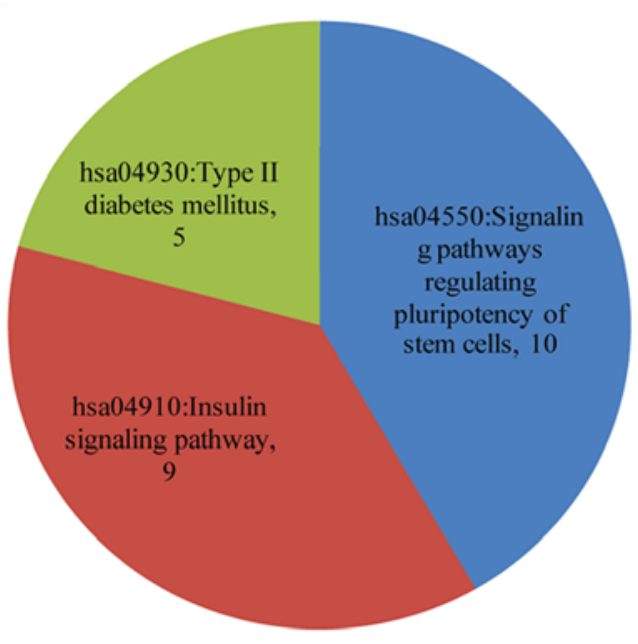

C

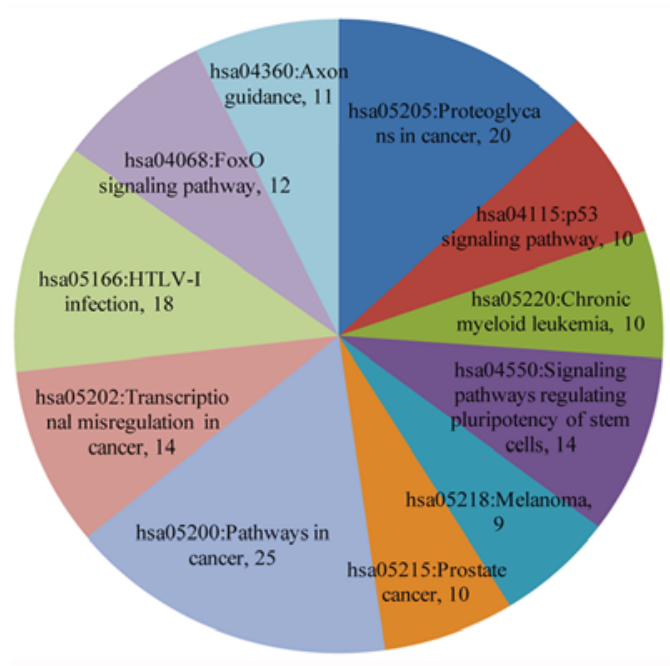

B
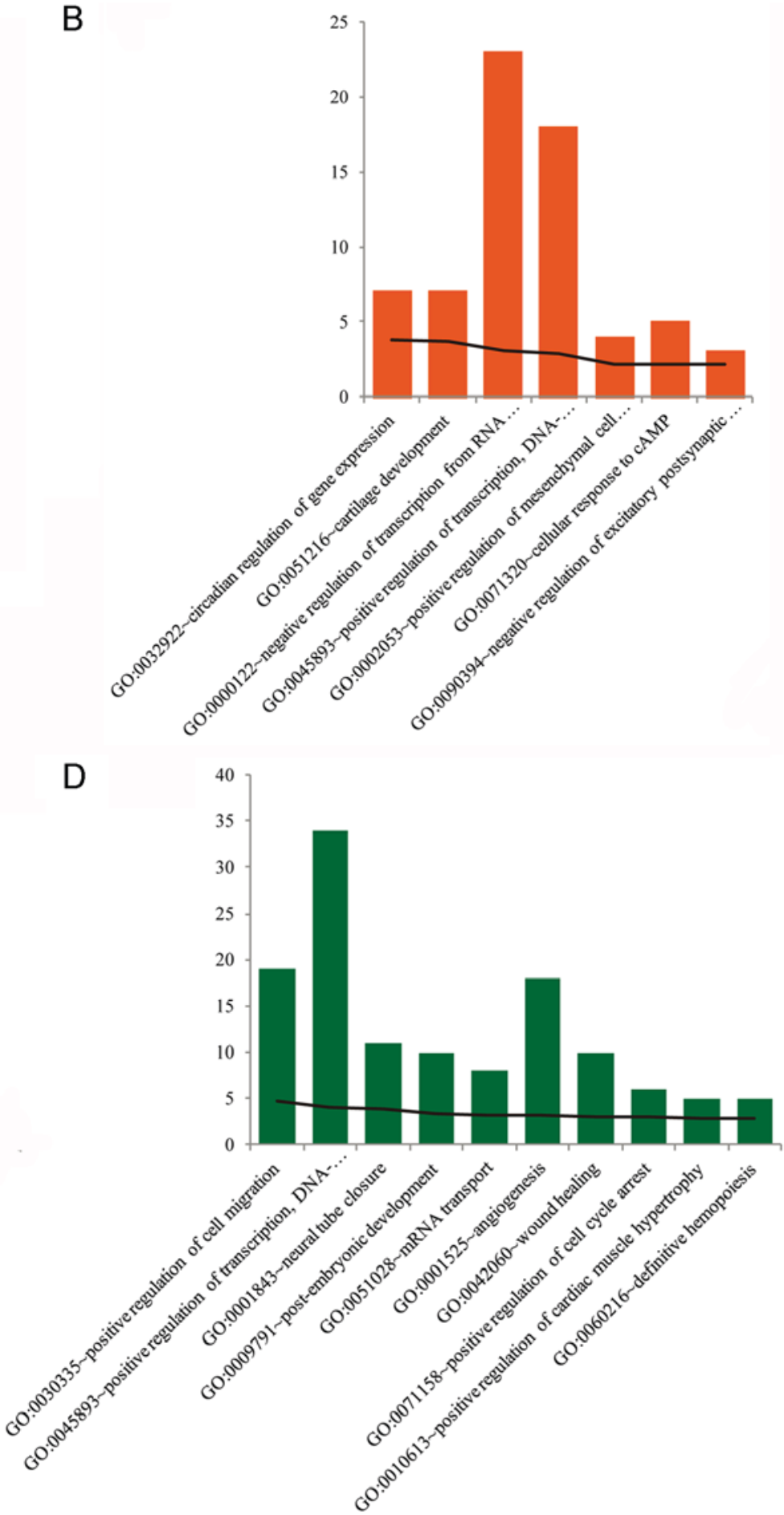

Figure 3. KEGG pathway enrichment and GO functional analyses on the target genes of differentially expressed miRNAs. (A) KEGG pathway results for target genes of upregulated miRNAs; (B) GO function results for target genes of upregulated miRNAs; (C) KEGG pathway results for target genes of downregulated miRNAs; and (D) GO function results for target genes of downregulated miRNAs. KEGG, Kyoto Encyclopedia of Genes and Genomes; GO, Gene Ontology.

acid type $\mathrm{B}$ receptor subunit 2 , and the genes identifying the focal adhesion pathway (hsa04510; $\mathrm{P}=5.74 \times 10^{-4}$ ) were $P I K 3 C G$, FMS-related tyrosine kinase $1, I G F 1$ and $K D R$.

\section{Discussion}

Although CRT has been established as a gold standard for LARC, the differences in CRT sensitivity between patients with LARC remain a challenge in clinical treatment (3).
In the present study, a total of 30 DEMs were identified between responders and non-responders in terms of sensitivity to CRT. Consistent with previous results (14), it was also identified that miR-375 and miR-148a were differentially expressed. In addition, a total of 11 miRNAs, including miR-371a-3p, exhibited significantly altered expressed in the miRNA-target gene network. These miRNAs were enriched in pathways including thiamine metabolism, signaling pathways regulating pluripotency of 


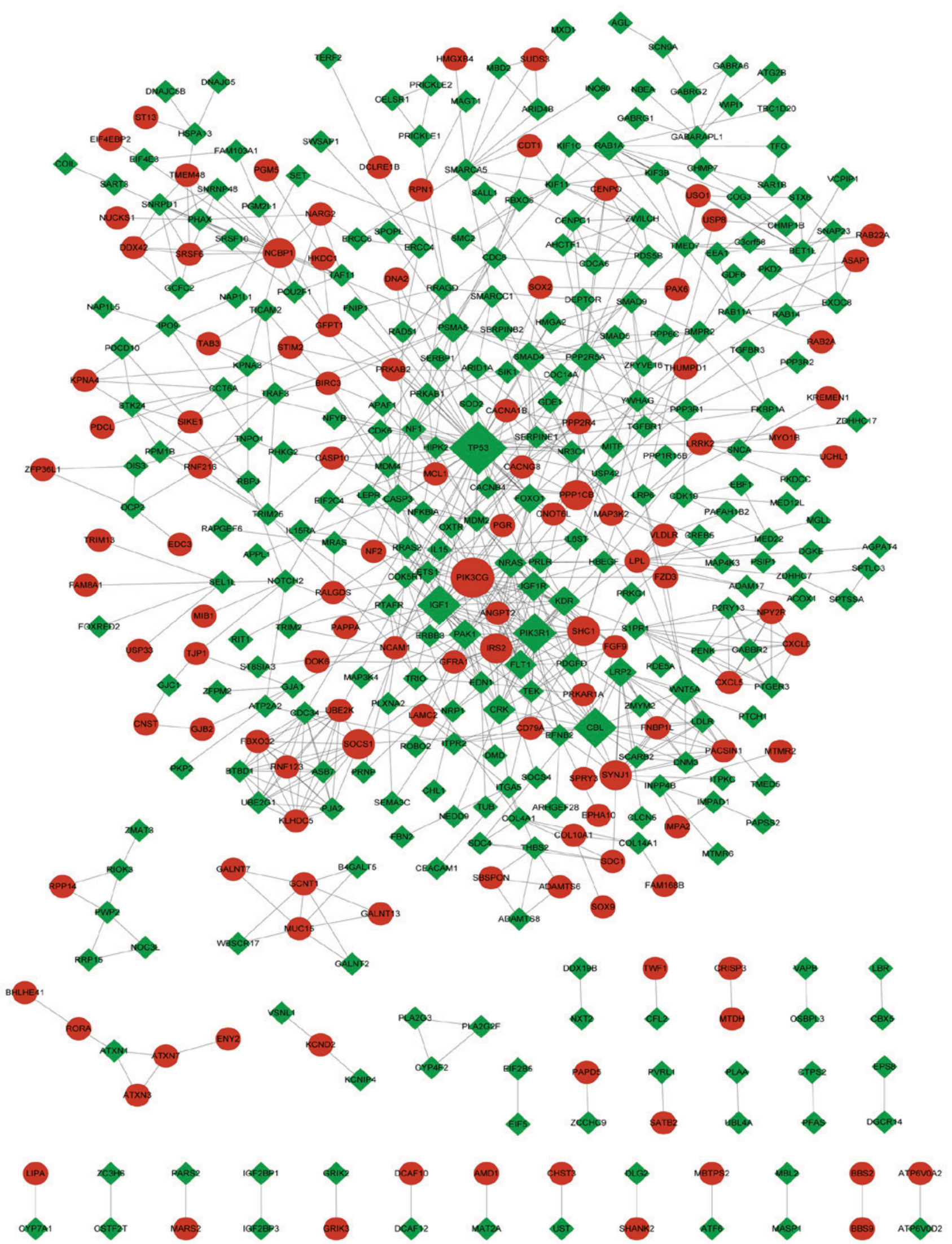

Figure 4. Protein-protein interaction network of target genes of differentially expressed microRNAs. Red circles represent upregulated genes and green diamonds represent downregulated genes. A larger node represents a higher degree of centrality.

stem cells and neuroactive ligand-receptor interaction. The target genes of these miRNAs were enriched in signaling pathways regulating pluripotency of stem cells and functions such as negative regulation of transcription from the 
A

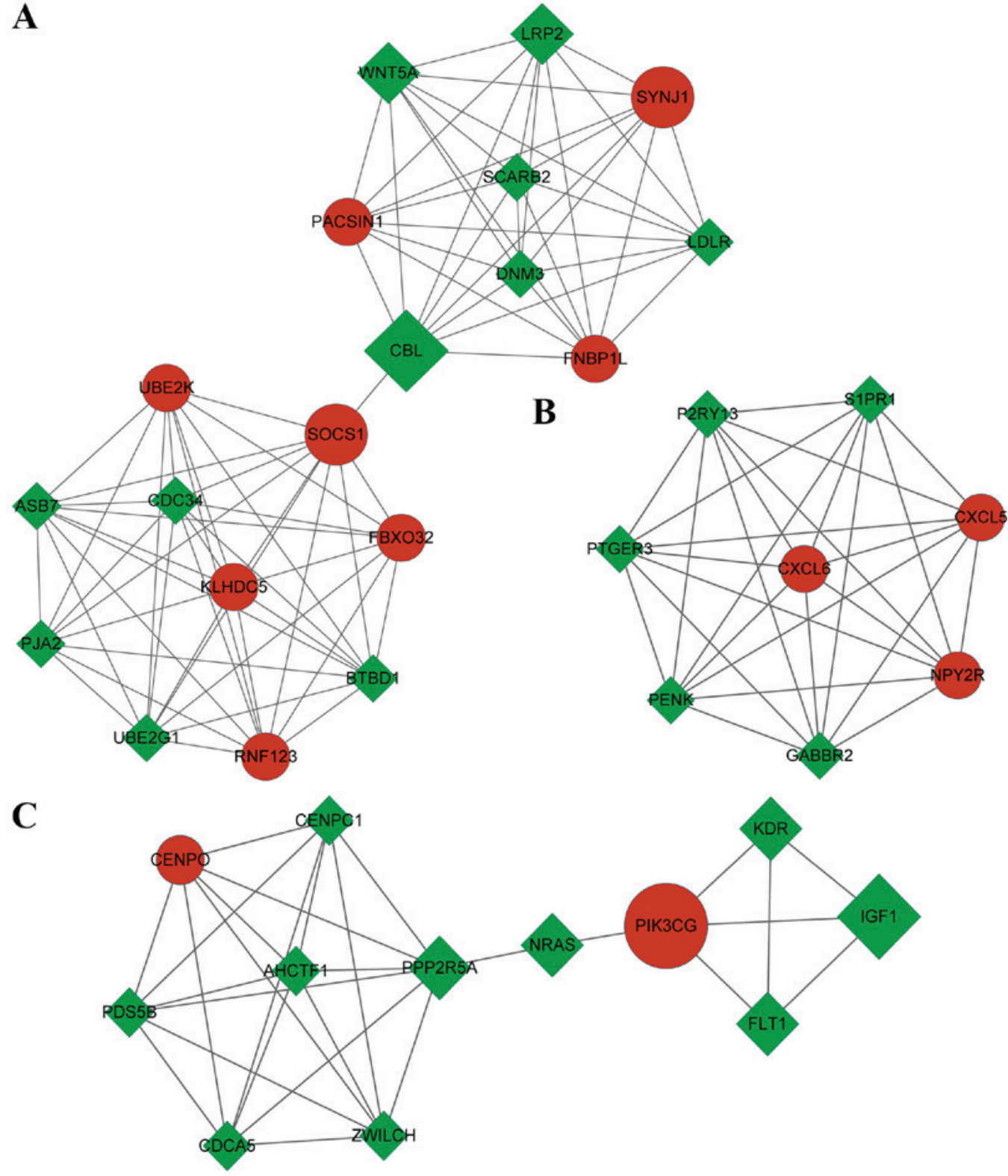

Figure 5. Three modules (A-C) extracted from the protein-protein interaction network. Red circles represent upregulated genes and green diamonds represent downregulated genes. A larger node represents a higher degree of centrality.

RNA polymerase II promoter. Furthermore, NRAS was revealed to be a key target gene of DEMs based on the PPI network, which was mainly enriched in functions such as intracellular signaling cascade.

miRNAs are involved in post-transcriptional gene regulation, and thus serve an essential role in numerous biological processes, including cancer cell proliferation $(28,29)$. miR-371a-3p has been associated with tumor cell proliferation (30). Although previous studies have indicated that serum miR-371a-3p may be a novel biomarker for tumor cells $(31,32)$, the association between miR-371a-3p and CRT sensitivity in LARC remains unknown. In the present study, the miRNA-target gene interaction analysis revealed that miR-371a-3p was downregulated. This result may indicate that miR-371a-3p is a potential factor for predicting CRT sensitivity in LARC. However, investigation of the mechanism of the effect of miR-371a-3p on CRT sensitivity is lacking. Notably, pathway analysis in the current study showed that thiamine metabolism was the pathway with the highest enrichment by miR-371a-3p. The role of thiamine in cancer is controversial according to previous studies. In one study, thiamine intake was inversely correlated with colon cancer risk (33). However, another study indicated that increased thiamine levels were beneficial for tumor cell survival, proliferation and chemotherapy resistance (34). Therefore, downregulated miR-371a-3p may affect CRT sensitivity in patients with LARC via the thiamine metabolism pathway.

Stem cells are responsible for maintaining differentiated cell numbers during normal physiology and at times of tissue stress (35). In CRT-treated patients with colorectal cancer, the upregulation of circulating cancer stem cell markers is vital for clinical parameters including CRT sensitivity (36). Hiroishi et al (37) indicated that prominin-1 and signal transducer CD24 contributed to the sensitivity of preoperative 
Table III. GO function and Kyoto Encyclopedia of Genes and Genomes pathway analysis for target genes associated with differentially expressed miRNAs in modules.

\begin{tabular}{|c|c|c|c|c|c|}
\hline Module & Identifier & Name & Count & P-value & Genes \\
\hline \multirow[t]{7}{*}{ A } & GO:0044265 & $\begin{array}{l}\text { Cellular macromolecule catabolic } \\
\text { process }\end{array}$ & 11 & $2.54 \times 10^{-9}$ & PJA2, RNF123, LDLR, BTBD1, UBE2K \\
\hline & GO:0009057 & Macromolecule catabolic process & 11 & $5.22 \times 10^{-9}$ & PJA2, RNF123, LDLR, BTBD1, UBE2K \\
\hline & GO:0019941 & $\begin{array}{l}\text { Modification-dependent protein } \\
\text { catabolic process }\end{array}$ & 10 & $7.51 \times 10^{-9}$ & PJA2, RNF123, BTBD1, UBE2K, UBE2G1 \\
\hline & GO:0043632 & $\begin{array}{l}\text { Modification-dependent } \\
\text { macromolecule catabolic process }\end{array}$ & 10 & $7.51 \times 10^{-9}$ & PJA2, RNF123, BTBD1, UBE2K, UBE2G1 \\
\hline & GO:0051603 & $\begin{array}{l}\text { Proteolysis involved in cellular } \\
\text { protein catabolic process }\end{array}$ & 10 & $1.11 \times 10^{-8}$ & PJA2, RNF123, BTBD1, UBE2K, UBE2G1 \\
\hline & hsa04120 & Ubiquitin-mediated proteolysis & 5 & $9.34 \times 10^{-5}$ & $U B E 2 K, U B E 2 G 1, S O C S 1, C B L, C D C 34$ \\
\hline & hsa04144 & Endocytosis & 3 & $4.84 \times 10^{-2}$ & $D N M 3, L D L R, C B L$ \\
\hline \multirow[t]{6}{*}{ B } & GO:0007186 & $\begin{array}{l}\text { G-protein-coupled receptor protein } \\
\text { signaling pathway }\end{array}$ & 8 & $2.67 \times 10^{-8}$ & P2RY13, S1PR1, PTGER3, PENK, CXCL5 \\
\hline & GO:0007166 & $\begin{array}{l}\text { Cell-surface receptor-linked signal } \\
\text { transduction }\end{array}$ & 8 & $9.06 \times 10^{-7}$ & P2RY13, S1PR1, PTGER3, PENK, CXCL5 \\
\hline & GO:0051350 & Negative regulation of lyase activity & 3 & $3.36 \times 10^{-4}$ & $S 1 P R 1, N P Y 2 R, G A B B R 2$ \\
\hline & GO:0031280 & $\begin{array}{l}\text { Negative regulation of cyclase } \\
\text { activity }\end{array}$ & 3 & $3.36 \times 10^{-4}$ & $S 1 P R 1, N P Y 2 R, G A B B R 2$ \\
\hline & GO:0007194 & $\begin{array}{l}\text { Negative regulation of adenylate } \\
\text { cyclase activity }\end{array}$ & 3 & $3.36 \times 10^{-4}$ & $S 1 P R 1, N P Y 2 R, G A B B R 2$ \\
\hline & hsa04080 & $\begin{array}{l}\text { Neuroactive ligand-receptor } \\
\text { interaction }\end{array}$ & 5 & $8.69 \times 10^{-5}$ & $P 2 R Y 13, S 1 P R 1, P T G E R 3, N P Y 2 R, G A B B R 2$ \\
\hline \multirow[t]{10}{*}{$\mathrm{C}$} & GO:0051301 & Cell division & 5 & $2.56 \times 10^{-5}$ & CENPO, PDS5B, AHCTF $1, Z W I L C H, C D C A 5$ \\
\hline & GO:0048754 & Branching morphogenesis of a tube & 3 & $8.01 \times 10^{-4}$ & $F L T 1, I G F 1, K D R$ \\
\hline & GO:0001763 & $\begin{array}{l}\text { Morphogenesis of a branching } \\
\text { structure }\end{array}$ & 3 & $1.04 \times 10^{-3}$ & $F L T 1, I G F 1, K D R$ \\
\hline & GO:0042127 & Regulation of cell proliferation & 5 & $1.13 \times 10^{-3}$ & NRAS, FLT1, PDS5B, IGF1, KDR \\
\hline & GO:0007059 & Chromosome segregation & 3 & $1.24 \times 10^{-3}$ & CENPO $, P D S 5 B, C D C A 5$ \\
\hline & hsa04510 & Focal adhesion & 4 & $5.74 \times 10^{-4}$ & $P I K 3 C G, F L T 1, I G F 1, K D R$ \\
\hline & hsa05214 & Glioma & 3 & $1.47 \times 10^{-3}$ & $P I K 3 C G, N R A S, I G F 1$ \\
\hline & hsa05218 & Melanoma & 3 & $1.87 \times 10^{-3}$ & PIK3CG, NRAS, IGF1 \\
\hline & hsa04370 & $\begin{array}{l}\text { Vascular endothelial growth factor } \\
\text { signaling pathway }\end{array}$ & 3 & $2.09 \times 10^{-3}$ & PIK3CG, NRAS, KDR \\
\hline & hsa05215 & Prostate cancer & 3 & $2.93 \times 10^{-3}$ & PIK3CG, NRAS, IGFI \\
\hline
\end{tabular}

GO, Gene Ontology; miRNAs, microRNAs; hsa, Homo sapiens.

CRT for LARC. In the present study, the GO functional analysis indicated that signaling pathways regulating the pluripotency of stem cells had the highest enrichment of upregulated miRNA-target genes, including PIK3CG and $A P C 2$. The product of PIK3CG is an modulator of extracellular signals and is vital for the maintenance of epithelia. A previous study indicated that downregulation of PIK3CG led to inhibition of the phosphoinositide 3-kinase/protein kinase $\mathrm{B}$ signaling pathway, and was associated with tumorigenesis and progression of colorectal cancer (38). Downregulation of $A P C 2$ may result in cancerous tumors, as $A P C 2$ regulates the uncontrolled growth of cells (39). The most common mutation in colon cancer is inactivation of APC (40). Thus, on the basis of the results of the present study, miRNA-target genes APC2 and PIK3CG may contribute to CRT sensitivity through signaling pathways regulating the pluripotency of stem cells.

$N R A S$ is an intracellular signal cascade mediator that initiates the mitogen-activated protein kinase signaling pathway (41). Mutation of NRAS is associated with progression of colorectal cancer (42). Although NRAS is an intracellular signal cascade mediator (41), the role of NRAS in CRT sensitivity in patients with LARC remains unknown. In the present study, target genes in the PPI network, including NRAS were enriched the intracellular signaling cascade pathway. This indicates that the differential expression of NRAS may serve 
an important role in determining CRT sensitivity in LARC via the intracellular signaling cascade.

The present study has a number of limitations. First, the sample size was small. Secondly, the present study used bioinformatic tools to filter and predict potential target genes which could affect the CRT response in LARC, but did not investigate the potential mechanism in vitro or in vivo. No experiments using clinical samples were performed. Lastly, previous studies have confirmed that mutations in genes for potential biomarkers, including KRAS proto-oncogene, BRAF proto-oncogene and NRAS, are associated with tumorigenesis and response to anti-epidermal growth factor receptor therapy in colorectal cancer (43-45). Next-generation sequencing to identify mutations in these potential biomarkers may elucidate the key mechanisms mediating CRT sensitivity in patients with LARC. Therefore, further clinical investigation based on a larger sample size and using high-throughput sequencing data is required to confirm the results of the present study.

In conclusion, downregulated miR-371a-3p may affect CRT sensitivity in patients with LARC via the thiamine metabolism pathway. Target genes including PIK3CG and $A P C 2$ may contribute to CRT sensitivity through signaling pathways regulating the pluripotency of stem cells. Furthermore, NRAS may serve an important role in determining CRT sensitivity in LARC via the intracellular signaling cascade.

\section{Acknowledgements}

Not applicable.

\section{Funding}

No funding was received.

\section{Availability of data and materials}

All data generated or analyzed during this study are included in this published article.

\section{Authors' contributions}

CL and CE were responsible for the conception and design of the research, drafting of the manuscript and performing the statistical analysis. YZ performed the data acquisition. WY performed the data analysis and interpretation. All authors read and approved the manuscript.

\section{Ethics approval and consent to participate}

Not applicable.

\section{Patient consent for publication}

Not applicable.

\section{Competing interests}

The authors declare that they have no competing interests.

\section{References}

1. WS Jr, Tarbell NJ, Yao M, Mehta MP and Wo JY: Rectal Cancer. John Wiley \& Sons, Inc, 2017.

2. Jomrich G, Silberhumer GR, Marian B, Beer A and Müllauer L: Programmed death-ligand 1 expression in rectal cancer. Eur Surg 48: 1-5, 2016

3. Kim JS, Jeong SY, Shin R, Oh HK, Park KJ and Park JG: 314 Preoperative versus postoperative chemoradiotherapy for locally advanced rectal cancer. Eur J Surg Oncol 38: 831-831, 2012.

4. Alderdice M, Dunne PD, Cole AJ, O'Reilly PG, McArt DG, Bingham V, Fuchs MA, McQuaid S, Loughrey MB, Murray GI, et al: Natural killer-like signature observed post therapy in locally advanced rectal cancer is a determinant of pathological response and improved survival. Mod Pathol 30: 1287-1298, 2017.

5. Sauer R, Becker H, Hohenberger W, Rödel C, Wittekind C, Fietkau R, Martus P, Tschmelitsch J, Hager E, Hess CF, et al: Preoperative versus postoperative chemoradiotherapy for rectal cancer. N Engl J Med 351: 1731-1740, 2004.

6. Cervantes A and Glynne-Jones R: Adjuvant chemotherapy for rectal cancer after preoperative radiation or chemoradiation: One size does not fit all. Ann Oncol 26: 617-619, 2015.

7. Bandres E, Agirre X, Bitarte N, Ramirez N, Zarate R, Roman-Gomez J, Prosper F and Garcia-Foncillas J: Epigenetic regulation of microRNA expression in colorectal cancer. Int J Cancer 125: 2737-2743, 2009.

8. Gaedcke J, Grade M,S $\varnothing$ kilde R, Kaczkowski B, Difilippantonio M, Ghadimi M, Ried T and Litman T: Abstract \#584: miRNA profiles predictive for response to chemoradiotherapy in rectal cancer. Cancer Res 69, 2009.

9. Svoboda M, Sana J, Fabian P, Kocakova I, Gombosova J, Nekvindova J, Radova L, Vyzula R and Slaby O: MicroRNA expression profile associated with response to neoadjuvant chemoradiotherapy in locally advanced rectal cancer patients. Radiat Oncol 7: 195, 2012.

10. Drebber U, Lay M, Wedemeyer I, Vallböhmer D, Bollschweiler E, Brabender J, Mönig SP, Hölscher AH, Dienes HP and Odenthal M: Altered levels of the onco-microRNA 21 and the tumor-suppressor microRNAs 143 and 145 in advanced rectal cancer indicate successful neoadjuvant chemoradiotherapy. Int J Oncol 39: 409-415, 2011.

11. Pradhan M, Ledford L, Pandit Y and Palakal M: Global analysis of miRNA target genes in colon rectal cancer. In: IEEE International Conference on Bioinformatics and Biomedicine, pp341-345, 2011.

12. Ju JA, Huang CT, Lan SH, Wang TH, Lin PC, Lee JC, Tian YF and Liu HS: Characterization of a colorectal cancer migration and autophagy-related microRNA miR-338-5p and its target gene PIK3C3. Biomarkers Genomic Med 5: 74-78, 2013.

13. Naccarati A, Pardini B, Stefano L, Landi D, Slyskova J, Novotny J, Levy M, Polakova V, Lipska L and Vodicka P: Polymorphisms in miRNA-binding sites of nucleotide excision repair genes and colorectal cancer risk. Carcinogenesis 33: 1346-1351, 2012.

14. Conde-Muino R, Cano C, Sanchez-Martin V, Herrera A, Comino A, Medina PP Palma P and Cuadros M: Preoperative chemoradiotherapy for rectal cancer: The sensitizer role of the association between miR-375 and c-Myc. Oncotarget 8: 82294-82302, 2017.

15. Smyth GK: Limma: Linear models for microarray data. In: Bioinformatics and Computational Biology Solutions Using $\mathrm{R}$ and Bioconductor. Gentleman R, Carey VJ, Huber W, Irizarry RA and Dudoit S (eds.) Springer New York, New York, NY, pp397-420, 2005.

16. Benjamini Y and Hochberg Y: Controlling the false discovery rate: A practical and powerful approach to multiple testing. J R Stat Soc Series B (Methodological): 289-300, 1995.

17. Dweep H and Gretz N: miRWalk2.0: A comprehensive atlas of microRNA-target interactions. Nat Methods 12: 697, 2015.

18. Dweep H, Gretz N and Sticht C: miRWalk database for miRNA-target interactions. Methods Mol Biol 1182: 289-305, 2014.

19. Betel D, Koppal A, Agius P, Sander C and Leslie C: Comprehensive modeling of microRNA targets predicts functional non-conserved and non-canonical sites. Genome Biol 11: R90, 2010.

20. Wong N and Wang X: miRDB: An online resource for microRNA target prediction and functional annotations. Nucleic Acids Res 43: D146-D152, 2015. 
21. Vejnar CE and Zdobnov EM: MiRmap: Comprehensive prediction of microRNA target repression strength. Nucleic Acids Res 40: 11673-11683, 2012.

22. Rigoutsos I, Miranda K and Huynh T: rna22: A unified computational framework for discovering miRNA precursors, localizing mature miRNAs, identifying 3'UTR Target-islands and determining the targets of mature-miRNAs. IBM Corporation, Yorktown Heights, NY, USA, 2007.

23. Agarwal V, Bell GW, Nam JW and Bartel DP: Predicting effective microRNA target sites in mammalian mRNAs. eLife 4 e05005, 2015.

24. Shannon P, Markiel A, Ozier O, Baliga NS, Wang JT, Ramage D, Amin N, Schwikowski B and Ideker T: Cytoscape: A software environment for integrated models of biomolecular interaction networks. Genome Res 13: 2498-2504, 2003.

25. Kanehisa M and Goto S: KEGG: Kyoto encyclopedia of genes and genomes. Nucleic Acids Res 28: 27-30, 2000.

26. Song W, Liu H, Wang J, Kong Y, Yin X and Zang W: MATHT: A web server for comprehensive transcriptome data analysis. J Theor Biol 455: 140-146, 2018.

27. Szklarczyk D, Franceschini A, Wyder S, Forslund K, Heller D, Huerta-Cepas J, Simonovic M, Roth A, Santos A, Tsafou KP, et al: STRING v10: Protein-protein interaction networks, integrated over the tree of life. Nucleic Acids Res 43: D447-D452, 2015

28. Catto JW, Alcaraz A, Bjartell AS, De Vere White R, Evans CP Fussel S, Hamdy FC, Kallioniemi O, Mengual L, Schlomm T and Visakorpi T: MicroRNA in prostate, bladder and kidney cancer: A systematic review. Eur Urol 59: 671-681, 2011.

29. Lu J, Getz G, Miska EA, Alvarez-Saavedra E, Lamb J, Peck D Sweet-Cordero A, Ebert BL, Mak RH, Ferrando AA, et al MicroRNA expression profiles classify human cancers. Nature 435: 834-838, 2005.

30. Ruf CG, Dinger D, Port M, Schmelz HU, Wagner W, Matthies C, Müller-Myhsok B, Meineke V and Abend M: Small RNAs in the peripheral blood discriminate metastasized from non-metastasized seminoma. Mol Cancer 13: 47, 2014.

31. Dieckmann KP, Radtke A, Spiekermann M, Balks T, Matthies C, Becker P, Ruf C, Oing C, Oechsle K, Bokemeyer C, et al: Serum levels of MicroRNA miR-371a-3p: A sensitive and specific new biomarker for germ cell tumours. Eur Urol 71: 213-220, 2017.

32. Spiekermann M, Belge G, Winter N, Ikogho R, Balks T, Bullerdiek J and Dieckmann KP: MicroRNA miR-371a-3p in serum of patients with germ cell tumours: Evaluations for establishing a serum biomarker. Andrology 3: 78-84, 2015.

33. Slattery ML, Potter JD, Coates A, Ma KN, Berry TD, Duncan DM and Caan BJ: Plant foods and colon cancer: An assessment of specific foods and their related nutrients (United States). Cancer Causes Control 8: 575-590, 1997.

34. Lu'o'ng KV and Nguyễn LT: The role of thiamine in cancer: Possible genetic and cellular signaling mechanisms. Cancer Genomics Proteomics 10: 169-185, 2013.
35. Buczacki S, Davies RJ and Winton DJ: Stem cells, quiescence and rectal carcinoma: an unexplored relationship and potential therapeutic target. Br J Cancer 105: 1253-1259, 2011.

36. Mirzaei A, Tavoosidana G, Modarressi MH, Rad AA, Fazeli MS Shirkoohi R, Tavakoli-Yaraki M and Madjd Z: Upregulation of circulating cancer stem cell marker, DCLK1 but not Lgr5, in chemoradiotherapy-treated colorectal cancer patients. Tumour Biol 36: 4801-4810, 2015

37. Hiroishi K, Inomata M, Kashima K, Yasuda K, Shiraishi N, Yokoyama S and Kitano S: Cancer stem cell-related factors are associated with the efficacy of pre-operative chemoradiotherapy for locally advanced rectal cancer. Exp Ther Med 2: 465-470, 2011.

38. Semba S, Itoh N, Ito M, Youssef EM, Harada M, Moriya T, Kimura W and Yamakawa M: Down-regulation of PIK3CG, a catalytic subunit of phosphatidylinositol 3-OH kinase, by $\mathrm{CpG}$ hypermethylation in human colorectal carcinoma. Clin Cancer Res 8: 3824-3831, 2002.

39. Jarrett CR, Blancato J, Cao T, Bressette DS, Cepeda M, Young PE, King CR and Byers SW: Human APC2 localization and allelic imbalance. Cancer Res 61: 7978-7984, 2001.

40. Yang D, Zhang M and Gold B: Origin of somatic mutations in $\beta$-catenin versus APC in colon cancer: Random mutagenesis in animal models versus Non-random mutagenesis in humans. Chem Res Toxicol 30: 1369-1375, 2017.

41. Hao T and Brooks TA: Abstract 2110: Modulating NRAS mRNA translation by nucleic acid clamp-mediated stabilization of the 5'-UTR G-quadruplex. Cancer Res 75: 2110, 2015.

42. Vaughn CP, Zobell SD, Furtado LV, Baker CL and Samowitz WS: Frequency of KRAS, BRAF, and NRAS mutations in colorectal cancer. Genes Chromosomes Cancer 50: 307-312, 2011.

43. Jauhri M, Bhatnagar A, Gupta S, Bp M, Minhas S, Shokeen Y and Aggarwal S: Prevalence and coexistence of KRAS, BRAF PIK3CA, NRAS, TP53, and APC mutations in Indian colorectal cancer patients: Next-generation sequencing-based cohort study. Tumour Biol 39: 1010428317692265, 2017.

44. Hsu HC, Thiam TK, Lu YJ, Yeh CY, Tsai WS, You JF, Hung HY, Tsai CN, Hsu A, Chen HC, et al: Mutations of KRAS/NRAS/BRAF predict cetuximab resistance in metastatic colorectal cancer patients. Oncotarget 7: 22257-22270, 2016.

45. Bagadi SB, Sanghvi M, Nair SB and Das BR: Combined mutational analysis of KRAS, NRAS and BRAF genes in Indian patients with colorectal carcinoma. Int J Biol Markers 27: 27-33, 2012.

This work is licensed under a Creative Commons Attribution-NonCommercial-NoDerivatives 4.0 International (CC BY-NC-ND 4.0) License. 